\title{
NEUROGENÉTICA EN EL PERÚ, EJEMPLO DE INVESTIGACIÓN TRASLACIONAL
}

\author{
Pilar Mazzetti ${ }^{1,2, a, c}$, Miguel Inca-Martínez $z^{1, b}$, Indira Tirado-Hurtado ${ }^{1, b}$, Karina Milla-Neyra ${ }^{1, b}$, \\ Gustavo Silva-Paredes ${ }^{1, e}$, Anastasia Vishnevetsky ${ }^{1,3,4, d}$, Mario Cornejo-Olivas ${ }^{1, a}$
}

\begin{abstract}
RESUMEN
La neurogenética es una disciplina emergente en el Perú que vincula la investigación básica con la práctica clínica. El Centro de Investigación Básica en Neurogenética, es el único centro en el Perú dedicado a la atención especializada de enfermedades neurogenéticas. La investigación en esta área está estrechamente ligada a la enfermedad de Huntington, desde la genotipificación del gen HTT por PCR, hasta los actuales estudios de haplogrupos en esta enfermedad. La investigación en otras enfermedades monogénicas permitió la implementación de metodologías alternativas para la genotipificación del síndrome $X$ frágil y distrofia miotónica tipo 1. Esfuerzos colaborativos nacionales e internacionales han permitido conocer nuevas variantes genéticas en enfermedades complejas, como la enfermedad de Parkinson y Alzheimer. El entrenamiento multidisciplinario y la mentoría fomentan la formación de nuevos especialistas en neurogenética, permitiendo el crecimiento sostenido de esta disciplina en el país. El impulso de la investigación en el Perú ha impulsado el crecimiento de la investigación en neurogenética; sin embargo, las limitaciones en infraestructura, tecnología y capacitación aún son un reto para el crecimiento de investigación en esta disciplina.
\end{abstract}

Palabras clave: Investigación traslacional; Neurología; Genética (fuente: DeCS BIREME).

\section{NEUROGENETICS IN PERU, EXAMPLE OF TRANSLATIONAL RESEARCH}

\begin{abstract}
Neurogenetics is an emerging discipline in Peru that links basic research with clinical practice. The Neurogenetics Research Center located in Lima, Peru is the only unit dedicated to the specialized care of neurogenetic diseases in the country. From the beginning, neurogenetics research has been closely linked to the study of Huntington's Disease (HD), from the PCR genotyping of the HTT gene, to the current haplogroup studies in HD. Research in other monogenic diseases led to the implementation of alternative methodologies for the genotyping of Fragile $X$ and Myotonic Dystrophy Type 1. Both, national and international collaborative efforts have facilitated the discovery of new genetic variants in complex multigenic diseases such as Parkinson's disease and Alzheimer's disease. Additionally, multidisciplinary education and mentoring have allowed for the training of new neurogenetics specialists, supporting the sustained growth of the discipline in the country. The promotion of research in Peru has spurred the growth of neurogenetics research, although limitations in infrastructure, technology, and education remain a challenge for the further growth of research in this field.
\end{abstract}

Key words: Translational research; Neurology; Genetics (source: MeSH NLM).

\section{NEUROGENÉTICA E INVESTIGACIÓN TRANSLACIONAL}

En el Perú, las enfermedades raras o huérfanas son un problema de salud pública pese a existir un subregistro sistemático de los pacientes que las padecen (1). Muchas de estas enfermedades corresponden a síndromes heredodegenerativos que afectan el sistema nervioso. La neurogenética, encargada del estudio de estas enfermedades es una disciplina que vincula inherentemente la investigación básica y la práctica clínica. Este vínculo entre el laboratorio y "la cama del hospital", conocido como investigación traslacional, facilita la aplicación de ideas, nuevos

\footnotetext{
Centro de Investigación Básica en Neurogenética, Instituto Nacional de Ciencias Neurológicas, Lima, Perú.

Facultad de Medicina Humana, Universidad Nacional Mayor de San Marcos. Lima, Perú.

Northern Pacific Global Health Research Fellows Training Consortium. Bethesda, Maryland, EE. UU.

Perelman School of Medicine at the University of Pennsylvania. Philadelphia, EE. UU.

Médico neurólogo; ${ }^{\text {b. }}$ biólogo genetista; ${ }^{c}$ magíster en Gestión Pública; ${ }^{\text {d. }}$ bachiller en Neurociencias; ${ }^{\text {e. }}$ magíster en Salud Pública Internacional Recibido: 14-09-15 Aprobado: 18-11-15
} 
conceptos, y descubrimientos generados a través de la investigación básica, para el tratamiento o prevención de enfermedades neurogenéticas ${ }^{(2,3)}$. La mayoría de estas enfermedades, que no cuentan con tratamientos curativos disponibles, requieren el apoyo de un laboratorio de biología molecular para lograr diagnósticos específicos; así como un abordaje multidisciplinario. Es por ello, que la investigación básica adquiere un rol trascendental en la búsqueda de blancos terapéuticos potenciales, que mantengan la esperanza de encontrar posibles tratamientos para las personas afectadas y sus familias ${ }^{(4)}$.

\section{DESARROLLO DE LA NEUROGENÉTICA EN EL PERÚ}

El Centro de Investigación Básica en Neurogenética (CIBN) del Instituto Nacional de Ciencias Neurológicas (INCN), es el único centro de investigación dedicado a la neurogenética en el Perú. Su historia se remonta al esfuerzo de una médica neuróloga e ingeniera química que iniciaron las atenciones de estas patologías. Sin embargo, no fue hasta el 24 de abril de 1995 que es reconocida oficialmente como Unidad de Neurogenética, en conmemoración a la fecha de la primera extracción de ADN realizada en esta unidad. Para el año 2000 se perfeccionaron las técnicas de extracción de ADN, gracias al apoyo de investigadores del Institut National de la Santé et de la Recherche Médicale de Francia, y se recibió el primer termociclador, donado por la Embajada de Japón, marcando el inicio de los procedimientos de amplificación de ADN mediante técnicas de reacción en cadena de la polimerasa o PCR (disponible en www. incngen.org.pe). A partir de ese mismo año, se realizó la genotipificación del microsatélite CAG en el gen HTT, responsable de la enfermedad de Huntington (EH). Posterior a ello, se han venido implementando, en el contexto de investigación, otras pruebas moleculares vinculadas a enfermedades neurogenéticas tales como la ataxia espinocerebelosa tipo 3 o SCA3/MJD, la enfermedad de Kennedy, el síndrome $X$ frágil, la distonía primaria DYT1, la distrofia miotónica tipo 1, (DM1) o enfermedad de Steiner, entre otros ${ }^{(5)}$. El trabajo coordinado entre la clínica y laboratorio, la producción científica sostenida, así como la consulta estandarizada ${ }^{(6)}$ motivaron el reconocimiento de la Unidad como Centro de Investigación Básica en Neurogenética desde octubre de $2011^{\text {(7) }}$ (Figura 1).

\section{ENFERMEDAD DE HUNTINGTON, UNA ENFERMEDAD NEUROGENÉTICA PREVALENTE EN EL PERÚ}

La neurogenética en el Perú está estrechamente ligada a la investigación de la enfermedad de Huntington. Los primeros reportes de EH en el país se remontan a 1950, cuando Saavedra et al. comunicaron el caso de una familia afectada procedente de Cotahuasi ${ }^{(8)}$. Desde 1983 se han publicado diversas descripciones que destacan la incidencia elevada de EH en un valle al sur de Lima, sugiriendo que esta región sería el principal foco de $\mathrm{EH}$ en el Perú (9). Un estudio reciente sobre la distribución de los casos de $\mathrm{EH}$, muestra que aún hay un número significativo de casos en este valle; sin embargo, la frecuencia de los casos se ha incrementado no solo en Lima, sino también en las regiones norte y sur del país (10); incluso se han descrito casos de $\mathrm{EH}$ en comunidades nativas de la selva amazónica peruana (11). Este conocimiento ha sido la base para la implementación de programas de atención neurogenética especializada en favor de los pacientes y familias de estas regiones.

Diversos estudios colaborativos nacionales e internacionales han contribuido a un mejor conocimiento de EH en población peruana; como las descripciones clínicas y moleculares de fenotipos clínicos de EH, incluyendo la forma clásica del adulto, las formas juveniles

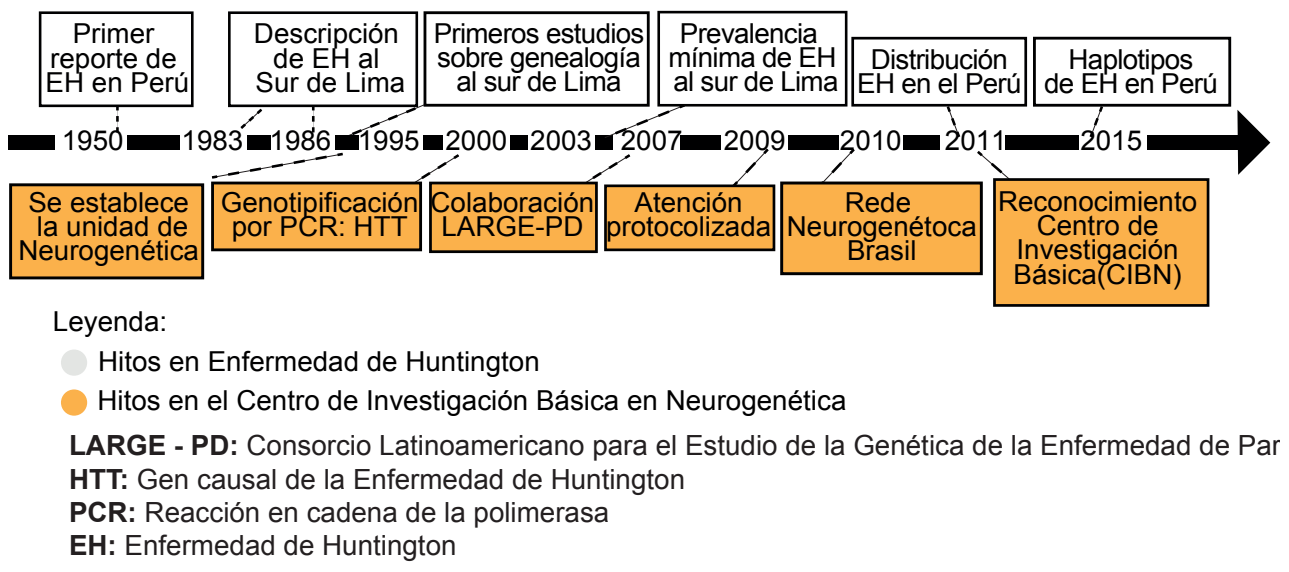

Figura 1. Cronología de la Neurogenética en el Perú 
y los casos de presentación tardía (12);obtenidas a través de registros sistematizados locales que apoyarían iniciativas internacionales como el estudio observacional ENROLL-HD (https://www.enroll-hd.org/), para facilitar la ejecución de ensayos clínicos de potenciales nuevos tratamientos para $\mathrm{EH}$. Asimismo, se han identificado los haplogrupos del gen HTT asociados a los cromosomas $\mathrm{EH}$, que permitirán el desarrollo de dianas terapéuticas específicas que supriman selectivamente el transcrito HTT expandido (13).

\section{INVESTIGACIÓN EN OTRAS ENFERMEDADES MONOGENICAS Y DESORDENES COMPLEJOS}

La investigación de otras enfermedades neurogenéticas monogénicas es emergente en el Perú. Las ataxias hereditarias dominantes poseen una distribución variable alrededor del mundo, siendo la SCA3/MJD la más frecuente ${ }^{(14)}$. Para el 2011, reportamos que solo 2 de 37 familias con diagnóstico clínico de ataxia familiar se encontraban afectadas con SCA3/MJD en el Perú (15). Desde el 2010, gracias a la colaboración con la Rede Neurogenética de Brasil (http://www. ufrgs.br/redeneurogenetica/), red de colaboración latinoamericana para el estudio de ataxias hereditarias, se viene estudiando hasta ocho ataxias dominantes, encontrándose que la ataxia espinocerebelosa tipo 10 o SCA10 es la ataxia dominante más frecuente en el Perú (16), contrastando con la distribución de ataxias dominantes a nivel mundial ya referida. El diagnóstico molecular de SCA10 en estas familias permite un mejor asesoramiento genético para limitar daños y prevenir complicaciones, como las crisis epilépticas asociadas a este desorden. Otras enfermedades neurodegenerativas como las distonías primarias, comparten fenotipos semejantes y requieren siempre un diagnóstico molecular confirmatorio. El diagnóstico genético distonía primaria DYT1, basado en la detección del a dirección del gen TORSIN 1A, ha permitido confirmar este diagnósticos en $20 \%$ de los casos analizados ${ }^{(17)}$

Se han desarrollado técnicas alternativas costoeficientes para el diagnóstico del síndrome $X$ frágil y la enfermedad de Steinert. El síndrome $X$ frágil, la causa más común de discapacidad intelectual heredada, es causado por la expansión del triplete CGG en el gen FMR1 en el cromosoma $X^{(18)}$. La técnica estándar de oro para el diagnóstico molecular de esta enfermedad, no disponible en el Perú, está basada en Southern Blot. En el CIBN se ha desarrollado un método alternativo basado en PCR de tiempo final empleando modificaciones con bisulfito, para amplificar microsatélites con alto contenido de GC ${ }^{(19)}$. Esta nueva propuesta permitiría identificar de forma indirecta el alelo premutado y la mutación completa vinculada al $\mathrm{X}$ frágil y sus síndromes asociados. Asimismo, el diagnóstico de la DM1, la miopatía hereditaria del adulto más frecuente, se realiza también empleando la técnica de Southern Blot. La implementación en el CIBN de técnicas alternativas basadas en PCR y sus modificaciones, han permitido apoyar el diagnóstico de los pacientes con DM1 con técnicas menos laboriosas, con precisión y robustez, semejantes a la técnica estándar de oro ${ }^{(20)}$.

La genética de las enfermedades complejas, como la enfermedad de Parkinson (EP), tiene también un desarrollo trascendental en el Perú. Las investigaciones en los aspectos genéticos de la EP se han realizado a través de estudios colaborativos nacionales, y en redes de colaboración internacionales. El Consorcio Latinoamericano para la Investigación en la Genética de la Enfermedad de Parkinson o LARGE-PD, por sus siglas en inglés, del cual el Perú es un miembro muy activo, viene promoviendo la investigación en esta área (21), junto con varios países latinoamericanos.

Entre los hallazgos más importantes de la genética de EP en el Perú se destacan: 1). La mutación G2019S en el gen $L R R K 2$, considerada la mutación dominante más frecuente en población caucásica, fue reportada con muy baja frecuencia en una población de pacientes con EP peruanos ${ }^{(22) ;}$ 2). El hallazgo inesperado de la alta frecuencia del polimorfismo Q1111H en el gen LRRK2 en población mestiza peruana, permitió desestimar este polimorfismo como un factor de riesgo para EP (23); 3). El descubrimiento de potenciales nuevas mutaciones asociadas a EP familiar, como la encontrada en tres hermanos portadores en el gen PARK2 en tres hermanos prtadores ${ }^{(24)}$; y 4). La descripción del caso de una mujer peruana de edad avanzada asintomática, portadora de la mutación $\mathrm{R} 1441 \mathrm{G}$ en el gen $L R R K 2$, que apoya la hipótesis de una penetrancia variable de esta mutación (25). Estos hallazgos en población peruana colaboran con los esfuerzos a nivel mundial en la búsqueda de nuevas variantes genéticas causales o de riesgo para EP.

Otro factor genético relacionado a enfermedades neurodegenerativas, y que viene siendo estudiado en Perú, es el alelo $\varepsilon 4$ del gen de la apolipoproteína $\mathrm{E}$ o $A P O E$. Este alelo ha sido relacionado con diversos desórdenes neurodegenerativos como la enfermedad de Alzheimer (EA) y la EP. Los estudios de Marca et al. en $A P O E$, en población sana mestiza peruana, sugieren un baja frecuencia del alelo $\varepsilon 4$ en nuestra población (26); mientras que los estudios en pacientes afectados con EP y controles sanos desestiman la asociación del alelo $\varepsilon 4$ como un factor de riesgo para EP (27). Sin embargo, no se descarta la posibilidad de asociación con endofenotipos de esta enfermedad. Asimismo, se ha descrito la primera familia peruana con EA de inicio temprano, asociado a 
alteraciones en el gen de la presenilina 1 (PSEN1); este estudio incluyó un análisis de ancestría que permitió sugerir un posible origen amerindio de algunas variantes nuevas encontradas en las regiones circundantes a la mutación causante de esta forma de inicio temprano de $\mathrm{EA}^{(28)}$.

\section{FORMACIÓN DE REDES \\ INTEGRADAS DE INVESTIGADORES NACIONALES E INTERNACIONALES, UNA NECESIDAD EN NEUROGENETICA}

El trabajo en equipo y el intercambio multidisciplinario de conocimientos, permiten desarrollar investigación que aborda de manera holística las necesidades de los pacientes, permitiendo la formación integral de investigadores en el Perú. La necesidad de atención integral de los pacientes con enfermedades neurogenéticas ha facilitado el trabajo conjunto de investigadores con formación en varias áreas como las neurociencias, la biología y la genética, muchos de ellos en diferentes etapas de formación como estudiantes, de pre y posgrado nacionales, e investigadores visitantes de otros países.

Las adaptaciones innovadoras y costo-eficientes realizadas en los países en vías de desarrollo, como el Perú, pueden ser compartidas con otros países que todavía mantienen limitaciones de acceso a varios servicios. Los investigadores invitados, varios de ellos extranjeros, encuentran en la neurogenética peruana muchas oportunidades de aprendizaje en genética humana, fisiopatología, epidemiologia, práctica clínica, bioética, así como el impacto social de estas enfermedades. La concentración de las enfermedades neurogenéticas en un mismo centro especializado facilita el estudio de estas enfermedades, que en otros países sería posible solo con la participación y coordinación de varios centros y hospitales. Asimismo, los investigadores invitados aportan significativamente a la investigación en neurogenética, brindando otras estrategias de atención para enfermedades raras, y compartiendo conocimientos sobre técnicas y procedimiento de vanguardia.

La mentoría o mentoring es una estrategia de formación científica fundamental de investigación en salud ${ }^{29)}$, y está siendo aplicada con éxito en el entrenamiento en Neurogenética en el Perú. El modelo de mentoría vincula a investigadores experimentados con jóvenes investigadores que recién se inician en esta área. Este modelo promueve la formación de una masa crítica de científicos, permitiendo un crecimiento sostenido de esta disciplina en el país (Figura 2).

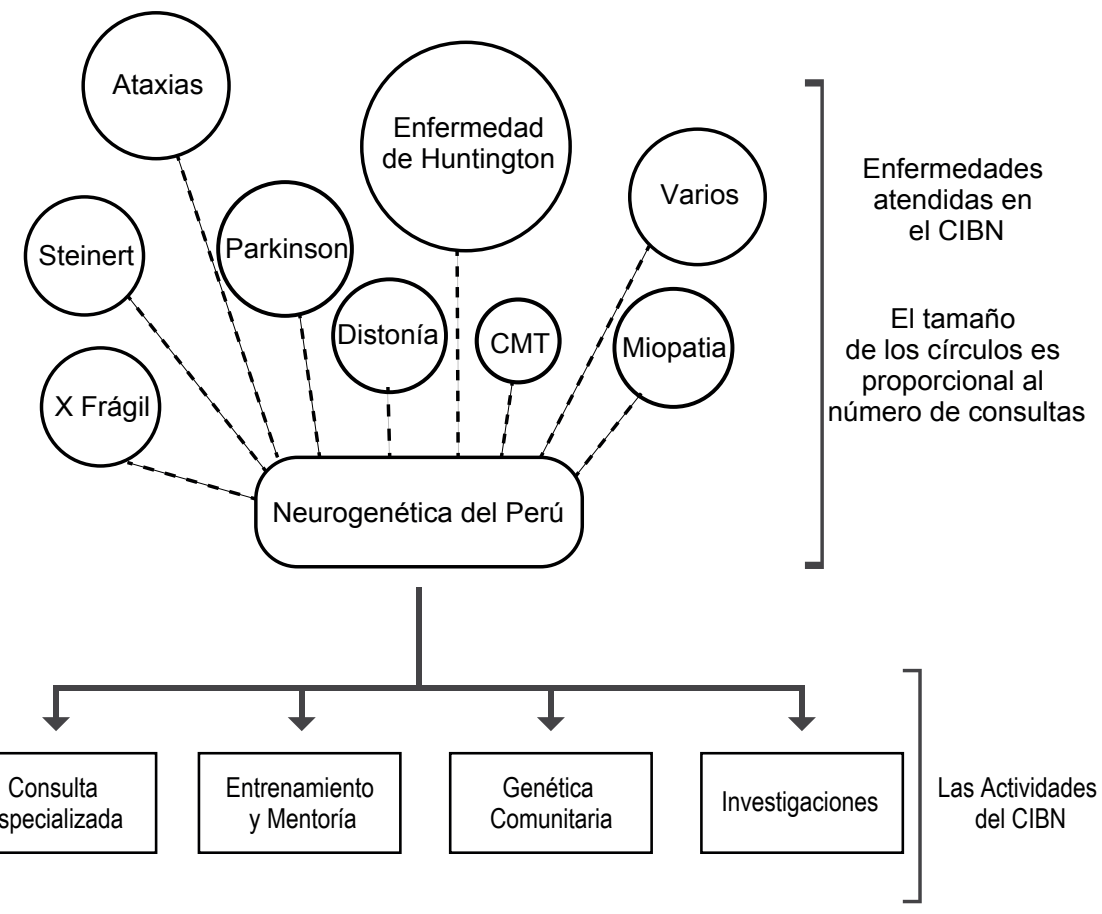

Figura 2. Enfermedades y servicios en el CIBN 


\section{NEUROGENÉTICA COMUNITARIA E INVESTIGACIÓN EN GENÉTICA DE POBLACIONES}

Los servicios que brindan atención en genética humana están localizados preferentemente en la capital y concentrados en 3 de los 8 Institutos Nacionales de Salud del Perú: el Instituto del Niño, Instituto de Enfermedades Neoplásicas y el INCN(30). Los bajos recursos de muchas de las familias afectadas no les permiten trasladarse hasta los centros especializados de la capital; ello genera la necesidad de cubrir la atención de enfermedades raras, como las enfermedades neurogenéticas, en otras regiones del país. Ello plantea el facilitar la accesibilidad a estos servicios y la telemedicina es una alternativa para ello, por lo que el CIBN ha establecido una red neurogenética incipiente a través de colegas interesados en varias regiones del Perú, que va a encontrar una oportunidad de estructurarse mejor al incorporarse al Sistema de Telesalud del Instituto de Gestión de Servicios de Salud.

Estrategias de genética comunitaria, como campañas de salud para enfermedades raras en lugares de alta incidencia de casos en el país, vienen siendo desarrolladas en el Perú en respuesta a la demanda creciente de atención especializada para la $\mathrm{EH}$ al sur de la capital. Otras estrategias comunitarias, como el estudio de poblaciones minoritarias en estricto respeto a los principios bioéticos, demuestran la importancia de considerar la variabilidad genética poblacional en estudio de asociación genética a nivel mundial $y$, al mismo tiempo, colaborar con el conocimiento del bagaje genético de estas poblaciones.

La investigación sobre la $\mathrm{EH}$ ha hecho ver que se requiere llevar la atención especializada a donde es más necesaria. A partir de ello, se ha firmado un convenio marco y otro específico tanto con la Dirección Regional de Salud de Lima Provincias como con la Microrred CañeteYauyos involucrando al centro de Salud Nuevo Imperial, con la finalidad de proporcionar asistencia técnica y conocimientos al personal de salud del referido Centro, para que ellos puedan atender mejor a las familias con EH que radican en la zona. Se realizan visitas periódicas en las que el personal del CIBN y el personal del Centro de Salud atienden en forma sistemática y ordenada a las familias afectadas. Ello también ha propiciado una serie de preguntas de investigación por parte del CIBN y, especialmente, del personal de salud de Cañete, quienes están guiando nuevas propuestas de investigación en base a las observaciones y realidad local.

En neurogenética, los resultados que se obtienen adquieren valor real en el contexto de la composición y evolución del genoma de nuestra población. EI INCN ha establecido un convenio con la Universidad del Altiplano, que busca generar capacidad local, el desarrollo de investigación e investigadores en Puno.

\section{LA INVESTIGACIÓN EN NEUROGENÉTICA EN EL PERÚ, RETOS Y PRÓXIMOS PASOS}

El desarrollo de la neurogenética en Perú en los últimos 20 años ha sido fructífera; sin embargo, aún presenta muchos desafíos. La obtención de financiamiento seguro y estable es un reto para los investigadores en el área de la genética molecular en el país, donde hay muchos problemas de salud prevalentes, y donde las prioridades cambian a través de tiempo. Una estrategia financiera es combinar financiamiento estatal dependiente del Ministerio de Salud con financiamiento externo de otras entidades, a través de fondos concursables. La ley de enfermedades raras en el Perú declara que enfermedades huérfanas, como las enfermedades neurogenéticas, son una prioridad para el gobierno; sin embargo, no está aún reglamentada ni cuenta con un financiamiento estructurado. Es por ello que la investigación en neurogenética en el Perú continúa careciendo de tecnologías de última generación y de infraestructura adecuada para apoyar la investigación en desarrollo. Además, la escasez de recursos humanos limita la posibilidad de conformar un equipo de atención integral, requiriendo profesionales especializados en asesoramiento genético, servicio social, nutrición, rehabilitación entre otros.

La investigación en $\mathrm{EH}$ es un claro ejemplo del desarrollo de la neurogenética en el Perú y tiene aún retos pendientes en nuestro país: 1). Un estudio sobre la ética y retos de diagnóstico presintomatico en $\mathrm{EH}$, una necesidad pendiente y clamorosamente solicitada por las familias de nuestros pacientes; 2). Un estudio sobre la calidad de vida de los pacientes y cuidadores en $\mathrm{EH}$; 3.) Un programa y protocolo de asesoramiento genético para $\mathrm{EH}$, basada en la información obtenida en los dos estudios anteriores; 4). Un programa de telemedicina en Cañete; 5). El apoyo para la conformación de una asociación de familias afectadas por $\mathrm{EH}$; y 6). El desarrollo de un programa nacional de atención integral para la $\mathrm{EH}$ y otras enfermedades neurogenéticas. El desarrollo de investigaciones colaborativas nacionales e internacionales, que busquen mejorar la calidad de atención de la $\mathrm{EH}$ y otras enfermedades raras, promoverán el desarrollo de nuevas líneas de investigación en el país. Así mismo, estas iniciativas mejorarán la calidad de los servicios de salud implementando mejores tratamientos a las personas afectadas con enfermedades raras y sus familias. 
Agradecimientos: NIH Research Training Grant \# R25 TW009345, por apoyo al entrenamiento de investigadores. A los biólogos genetistas Victoria Marca y Olimpio Ortega por sus valiosos comentarios a la versión final del manuscrito.

Contribuciones de autoría: PMS, MIM, ITH, KMN, GSP, AV y MCO participaron en la concepción y diseño del artículo, el análisis e interpretación de datos, redacción del artículo, revisión crítica del artículo y aprobación de su versión final.

Fuente de financiamiento: presupuesto de investigación del Instituto Nacional de Ciencias Neurológicas.

Conflictos de interés: los autores declaran no tener conflictos de interés en la publicación de este artículo.

\section{REFERENCIAS BIBLIOGRÁFICAS}

1. Ley que declara de interes nacional y preferente atencion el tratamiento de personas que padecen enfermedades raras o huerfanas, Pub, L. No. 29698 Lima, Perú: El Peruano (4 de junio de 2011).

2. WoolfSH. The meaning of translational research and why it matters. JAMA. 2008;299(2):211-3. doi: 10.1001/ jama.2007.26.

3. Ullastres C, Rendo D. Investigación Traslacional: Aprendiendo a escuchar Fundación Gaspar Casal. Madrid: Fundación Gaspar Casa; 2013.

4. Plenge RM, Scolnick EM, Altshuler D. Validating therapeutic targets through human genetics. Nat Rev Drug Discov. 2013;12(8):581-94. doi: $10.1038 /$ nrd4051.

5. Cornejo-Olivas M, Espinoza-Huertas K, Velit-Salazar MR, Veliz-Otani D, Tirado-Hurtado I, Inca-Martinez M, et al. Neurogenetics in Peru: clinical, scientific and ethical perspectives. J Community Genet. 2015;6(3):251-7. doi: 10.1007/s12687-015-0239-z.

6. Mazzetti PM, Ortega O, CornejoOlivas M, Torres G, Timana C. Guías de Procedimientos Médicos y Prácticas Clínicas de la Dirección Ejecutiva de Investigación, Docencia y Atención Especializada en Apoyo al Diagnóstico y Tratamiento. Lima: Instituto Nacional de Ciencias Neurológicas; 2010.

7. Creación e Implementación del Centro de Investigación Básica en Neurogenética. Resolución Directoral $\mathrm{N}^{\circ}$ 385-2011-INCN-DG. Lima: Ministerio de Salud (19 de octubre del 2011).

8. Saavedra A. Sobre un caso de Corea de Huntington. Rev Neuropsiquiatria. 1950;2:232-9.

9. Cuba JM. [A focus of Huntington's chorea in Peru]. Rev Neurol (Paris). 1986;142(2):151-3. [Article in French]
10. Lovaton-Espadin R, Timana CR, Alva GT, Cornejo MR, MarcaYsabel M, Ortega-Davila O, et al., editors. Huntington's disease in Peru: Spread of cases and Analysis of CAG repeats distribution. $60^{\text {th }}$ ASGH Annual meetings; 2010 November; Washington D.C., USA.

11. Cornejo-Olivas M, Mori N, Alva I, Zunt J, Mazzetti P. Huntington's Disease in an Indigenous Village in the Peruvian Amazon Jungle. In: Society IPaMD, editor. $16^{\text {th }}$ International Congress of Parkinson's Disease and Movement Disorders; 2012 June 1721; Dublin, Ireland.

12. Cornejo-Olivas MR, Inca-Martinez MA, Espinoza-Huertas K, VelizOtani D, Velit-Salazar MR, Marca V, et al. Clinical and molecular features of late onset huntington disease in a Peruvian cohort. J Huntingtons Dis. 2015;4(1):99-105.

13. Tirado I, Kay C, Cornejo-Olivas M, Collins JA, M. Ketelaar E, LindoSamanamud S, Inca-Martinez M, Ortega O, Marca V, Veliz-Otani D, Espinoza-Huertas K, Sotil G, Mazzetti P, Hayden MR. Determination of the origin of Huntington disease based on haplotypes in a Peruvian population. Annual Meeting of the American Society of Human Genetics; 2014 October 18-22; San Diego, California, USA.

14. Ruano L, Melo C, Silva MC, Coutinho P. The global epidemiology of hereditary ataxia and spastic paraplegia: a systematic review of prevalence studies. Neuroepidemiology. 2014;42(3):17483. doi: $10.1159 / 000358801$.

15. Cornejo-Olivas M, Chacón R, Marca V, Timana C, Flores M, Cosentino $\mathrm{C}$, et al. Frequency of spinocerebellar ataxia type 3 (SCA3) or Machado Joseph disease frequency in Peruvian population. $14^{\text {th }}$ International Congress Movement Disorders Society:
Internacional Parkinson Disease and Movement Disorders Society; 2011 June; Toronto, Canada.

16. Cornejo-Olivas M, Cornejo-Herrera I, Lindo-Samanamud $S$, Castilhos R, Saraiva-Pereira M, Jardim L, et al. Spinocerebellar Ataxia Type 10 or SCA10 in Peruvian Population. First Report of Three Families. Neurology. 2013;80.

17. Inca-Martinez $\mathrm{M}, \quad$ Ortega $\mathrm{O}$, Marca V, Lindo-Samanamud S, Espinoza-Huertas K, Veliz-Otani D, et al. Diagnóstico Molecular para Distonía Primaria DYT1: Diseño basado en PCR para la mutación 904_906delGAG/907 909delGAG en el gen TORSINA1A. En: XX Curso Internacional de Neurociencias. Lima, Perú, Instituto Nacional de Ciencias Neurológicas; 2014.

18. Biancalana V, Glaeser D, McQuaid $S$, Steinbach P. EMQN best practice guidelines for the molecular genetic testing and reporting of fragile $\mathrm{X}$ syndrome and other fragile $\mathrm{X}$-associated disorders. Eur J Hum Genet. 2015;23(4):417-25. doi: 10.1038/ejhg.2014.185.

19. Lindo-Samanamud S, Cornejo-Olivas M, Ortega O, Marca V, EspinozaHuertas K, Mazzetti P. Estrategia de genotipado del gen FMR1: Método de diagnóstico alternativo para el Síndrome X Frágil y otras enfermedades por expansión de trinucleótidos. Rev Med Hered. 2013;24(4):269-76.

20. Milla-Neyra K, Inca-Martinez M, Cornejo-Olivas M, Ortega O, Marca V, Lindo-Samanamud S, et al. Metodología basada en la combinación de las técnicas de PCR y TP-PCR para el diagnóstico molecular de la Distrofia Miotónica tipo 1 en el INCN. XXI Curso Internacional de Neurociencias; Lima, Perú, Instituto Nacional de Ciencias Neurológicas; 21 de junio del 2015. 
21. Foundation Psd [internet]. Linking PD Genes in a LARGE Way in Latin America [citado el 18 de noviembre del 2015]. Disponible en: http://www.pdf. org/fall14_spotlight

22. Mata IF, Cosentino C, Marca V, Torres L, Mazzetti P, Ortega O, et al. LRRK2 mutations in patients with Parkinson's disease from Peru and Uruguay. Parkinsonism Relat Disord. 2009;15(5):370-3. doi: 10.1016/j. parkreldis.2008.09.002.

23. Mata IF, Wilhoite GJ, Yearout D, Bacon JA, Cornejo-Olivas M, Mazzetti P, et al. Lrrk2 p.Q1111H substitution and Parkinson's disease in Latin America. Parkinsonism Relat Disord. 2011;17(8):629-31. doi: 10.1016/j. parkreldis.2011.05.003.

24. Cornejo-Olivas MR, Torres L, Mata IF, Mazzetti P, Rivas D, Cosentino C, et al. A Peruvian family with a novel PARK2 mutation: Clinical and pathological characteristics. Parkinsonism Relat Disord. 2015;21(5):444-8. doi: 10.1016/j.parkreldis.2015.01.005.
25. Cornejo-Olivas M, Torres L, Mazzetti P, Cosentino C, Zabetian CP, Mata IF. Variable penetrance of the LRRK2R1441G mutation in a Peruvian family [abstract]. In: Disorders m, editor. MDS Congress 20142013. p. 1154.

26. Marca V, Acosta O, CornejoOlivas M, Ortega O, Huerta D, Mazzetti P. [Genetic polymorphism of apolipoprotein E in a Peruvian population]. Rev Peru Med Exp Salud Publica. 2011 Dec;28(4):589-94. [Article in Spanish]

27. Marca V, Acosta O, Torres L, Ortega O, Cornejo-Olivas M, LIndoSamanamud $\mathrm{S}$, et al. Asociación entre el polimorfismo genético de la apolipoproteína E (ApoE) y la enfermedad de Parkinson. An Fac med. 2013;74(3):169-73. doi: http://dx.doi. org/10.15381/anales.v74i3.2629

28. Cornejo-Olivas MR, Yu CE, Mazzetti P, Mata IF, Meza M, LindoSamanamud S, et al. Clinical and molecular studies reveal a PSEN1 mutation (L153V) in a Peruvian family with early-onset Alzheimer's disease. Neurosci Lett. 2014;563:140-3. doi: 10.1016/j.neulet.2014.01.016.

29. Di Ruggiero E, Cohen JE, Cole DC, Forman L. Public health agenda setting in a global context: the International Labor Organization's decent work agenda. Am J Public Health. 2015;105(4):e58-61. doi: 10.2105/AJPH.2014.302455.

30. Cornejo-Olivas M, Velit-Salazar M, Avalos-Cruz T, Dueñas-Roque $M$, Inca-Martinez M, Mora-Alferez P, et al. Identification and characterization of Genetic Services in Peru. 62 $2^{\text {th }}$ Annual Meeting of the American Society of Human Genetics: American Society of Human Genetics; 2013 October; Boston, USA.

Correspondencia: Pilar Mazzetti

Dirección: Jr. Ancash 1271, Barrios Altos,

Lima 1, Perú.

Teléfono: (+511) 411-7779.

Correo electrónico:pilar:mazzetti.s@

incngen.org.pe

\section{Consulte la versión electrónica de la Revista Peruana de Medicina Experimental y Salud Pública en www.pubmed.gov}

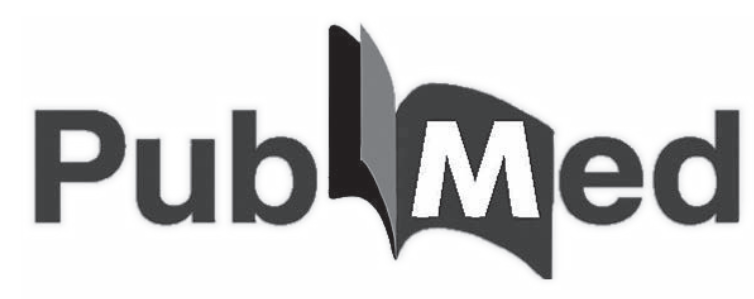

